\title{
Dirac Particle for the Position Dependent Mass in the Generalized Asymmetric Woods-Saxon Potential
}

\author{
Soner Alpdoğan and Ali Havare \\ Department of Physics, Mersin University, 33343 Mersin, Turkey \\ Correspondence should be addressed to Soner Alpdoğan; soneralpdogan@gmail.com
}

Received 14 April 2014; Accepted 19 June 2014; Published 10 July 2014

Academic Editor: Hong-Jian He

Copyright ( 2014 S. Alpdoğan and A. Havare. This is an open access article distributed under the Creative Commons Attribution License, which permits unrestricted use, distribution, and reproduction in any medium, provided the original work is properly cited. The publication of this article was funded by SCOAP S $^{3}$

\begin{abstract}
The one-dimensional Dirac equation with position dependent mass in the generalized asymmetric Woods-Saxon potential is solved in terms of the hypergeometric functions. The transmission and reflection coefficients are obtained by considering the onedimensional electric current density for the Dirac particle and the equation describing the bound states is found by utilizing the continuity conditions of the obtained wave function. Also, by using the generalized asymmetric Woods-Saxon potential solutions, the scattering states are found out without making calculation for the Woods-Saxon, Hulthen, cusp potentials, and so forth, which are derived from the generalized asymmetric Woods-Saxon potential and the conditions describing transmission resonances and supercriticality are achieved. At the same time, the data obtained in this work are compared with the results achieved in earlier studies and are observed to be consistent.
\end{abstract}

\section{Introduction}

The scattering and bound states in nonrelativistic and relativistic quantum mechanics within an external potential are studied in order to describe the behavior of particles, atoms, and molecules in physics. Thus, the nonrelativistic and relativistic particle equations in an external potential have been widely handled for the scattering and bound states [1-16]. In the recent years, these external potentials have been generalized to asymmetric potentials in theoretical and experimental physics [13-22]. Also, last year, an international team at the ISOLDE radioactive-beam facility at CERN reported that atomic nuclei could suppose asymmetric "pear" shapes [19].

Another important issue is the concept of position dependent mass. This concept is studied in different applications of physics, for example, electronic properties of semiconductor materials [23], quantum liquids [24], quantum dots [25], and the nuclear forces of relativistic two nucleons [26, 27].

In this paper, the generalized asymmetric Woods-Saxon potential (GAWSp), that includes the Woods-Saxon potential, is considered to obtain the solutions of the Dirac equation with position dependent mass. The Woods-Saxon potential
[28] has been widely used in the nonlinear theory of scalar mesons $[29,30]$, the nuclear shell model, and the distribution of nuclear densities [31,32]. The GAWSp is introduced in [16]

$$
V(x)=V_{0}\left[\frac{\theta(-x)}{q+p e^{-a(x+L)}}+\frac{\theta(x)}{\widetilde{q}+\widetilde{p} e^{b(x-\widetilde{L})}}\right] \text {, }
$$

where $\theta(x)$ is the Heaviside step function and $V_{0}$ is potential intensity and real. Parameters $L, p, q, \widetilde{L}, \widetilde{p}, \widetilde{q}, a$, and $b$ are real. The GAWSp is shown in Figure 1 and in special cases, the GAWSp turns into the potentials which are used in the nonrelativistic and relativistic quantum physics. Some special cases of the GAWSp are shown in Table 1.

The paper is organized as follows. In Section 2, the transmission and reflection coefficients are found for the effective mass Dirac particle. In Section 3, an equation is obtained for energy eigenvalues. In Section 4, the results and discussions and the plots constructed by using Mathematica Software are commented and the energy values are given numerically; moreover, the transmission resonance condition for the scattering states and supercriticality are derived and the data obtained in this work are compared with 
TABLE 1: The special potentials: the symmetric potentials and asymmetric (asy.) potentials (pot.).

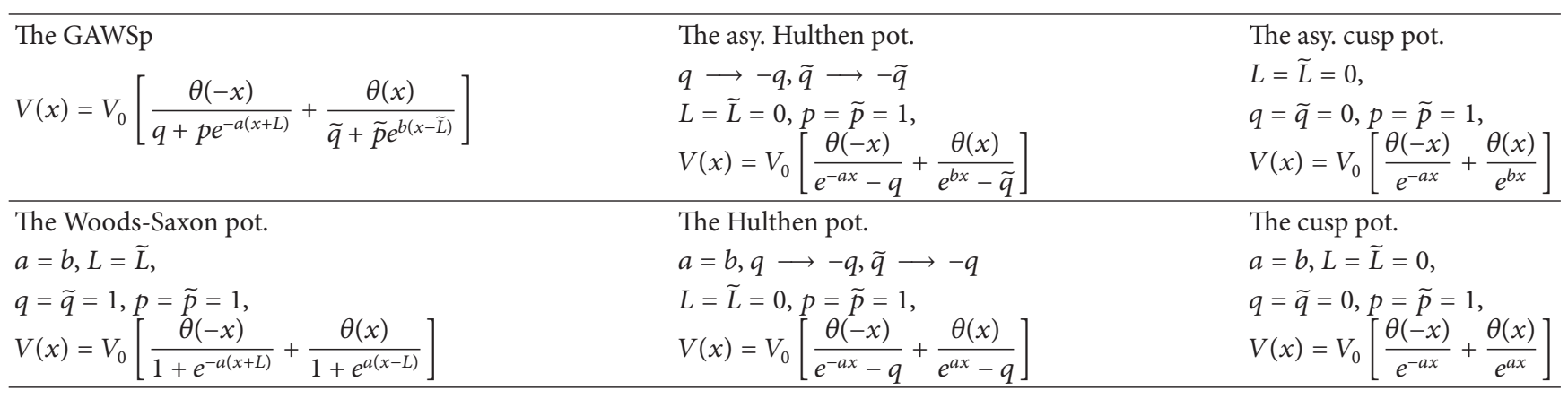

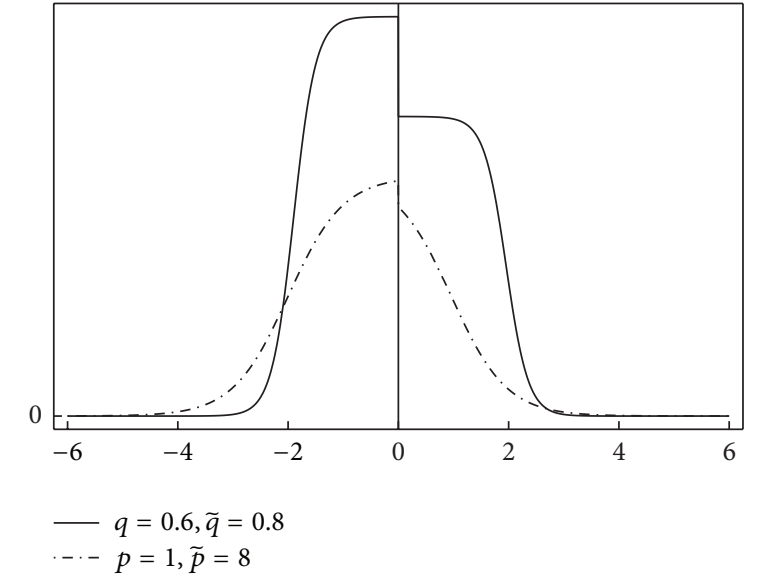

(a)

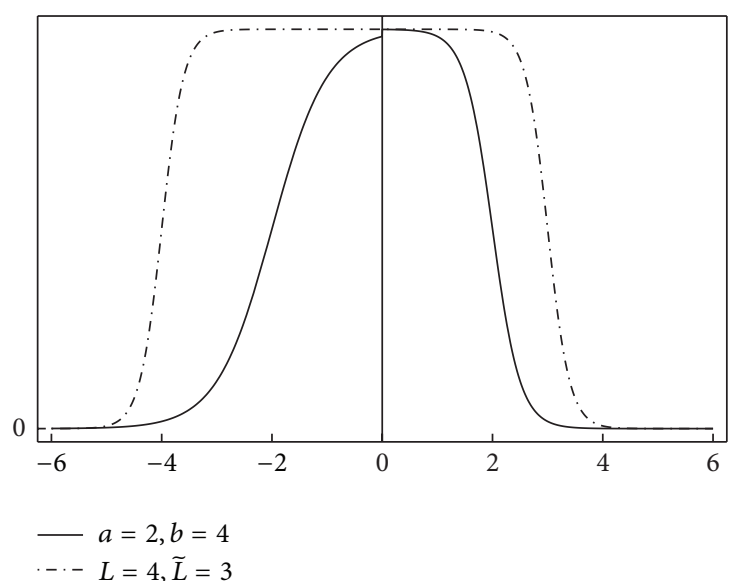

(b)

FIgURE 1: The different values of the GAWSp. (a) shows $V_{0}=1, a=5, b=5, p=1, \widetilde{p}=1, L=2$, and $\widetilde{L}=2$ (solid lines) and $V_{0}=1, q=1$, $\widetilde{q}=1, a=2, b=2, L=2$, and $\widetilde{L}=2$ (dash-dotted lines). (b) shows $V_{0}=1, p=1, \widetilde{p}=1, q=1, \widetilde{q}=1, L=2$, and $\widetilde{L}=2$ (solid lines) and $V_{0}=1, q=1, \widetilde{q}=1, a=5, b=5, p=1$, and $\widetilde{p}=1$ (dash-dotted lines).

the results achieved in earlier studies. Finally, the present paper is summarized in Section 5.

\section{Scattering Solutions of the GAWSp for the Variable Mass Dirac Particle}

The Dirac equation for the relativistic free-particle is given as the following form [20] (in natural units $\hbar=c=1$ ):

$$
\left[i \gamma^{\mu} \partial_{\mu}-m(x)\right] \Psi(x)=0,
$$

where $m(x)$, the Dirac particle mass, depends on one spatially coordinate $x$. In order to obtain the one-dimensional Dirac equation under an external potential $V(x)$, the gamma matrices $\gamma_{x}$ and $\gamma_{0}$ are reduced to the Pauli matrices $i \sigma_{x}$ and $i \sigma_{z}$, respectively,

$$
\begin{aligned}
& \left\{\left(\begin{array}{ll}
0 & 1 \\
1 & 0
\end{array}\right) \frac{d}{d x}-[E-V(x)]\left(\begin{array}{cc}
1 & 0 \\
0 & -1
\end{array}\right)+m(x)\left(\begin{array}{ll}
1 & 0 \\
0 & 1
\end{array}\right)\right\} \\
& \quad \times\left(\begin{array}{l}
U_{1}(x) \\
U_{2}(x)
\end{array}\right)=0,
\end{aligned}
$$

where $U_{1}(x)$ and $U_{2}(x)$ are decomposed into upper and lower components of the two-component wave function $\Psi(x)$ and (3) turns into the two coupled differential equations as follows:

$$
\begin{aligned}
& \frac{d U_{1}(x)}{d x}=-[m(x)+E-V(x)] U_{2}(x), \\
& \frac{d U_{2}(x)}{d x}=-[m(x)-E+V(x)] U_{1}(x) .
\end{aligned}
$$

Then the following two expressions are written as they were described by Flügge as [3]

$$
\begin{aligned}
& \Theta(x)=U_{1}(x)+i U_{2}(x), \\
& \Phi(x)=U_{1}(x)-i U_{2}(x)
\end{aligned}
$$

Putting these expressions into (4), the following equations are achieved:

$$
\begin{aligned}
& \frac{d \Theta(x)}{d x}=i[E-V(x)] \Theta(x)-i m(x) \Phi(x), \\
& \frac{d \Phi(x)}{d x}=-i[E-V(x)] \Phi(x)+i m(x) \Theta(x) .
\end{aligned}
$$


By using the two equations given above, for $\Theta(x)$ and $\Phi(x)$, the following two independently second-order differential equations are obtained:

$$
\begin{aligned}
& \frac{d^{2} \Theta(x)}{d x^{2}}-\frac{1}{m(x)} \frac{d m(x)}{d x} \frac{d \Theta(x)}{d x} \\
& +\left\{[E-V(x)]^{2}-m^{2}(x)+i \frac{d V(x)}{d x}\right. \\
& \left.+i[E-V(x)] \frac{1}{m(x)} \frac{d m(x)}{d x}\right\} \Theta(x)=0, \\
& \frac{d^{2} \Phi(x)}{d x^{2}}-\frac{1}{m(x)} \frac{d m(x)}{d x} \frac{d \Phi(x)}{d x} \\
& +\left\{[E-V(x)]^{2}-m^{2}(x)-i \frac{d V(x)}{d x}\right. \\
& \left.-i[E-V(x)] \frac{1}{m(x)} \frac{d m(x)}{d x}\right\} \Phi(x)=0 .
\end{aligned}
$$

The Dirac particle mass $m(x)$ is supposed in [20]

$$
m(x)=m_{0}+m_{1} f(x),
$$

where the function $f(x)$ is given by $f(x)=\theta(-x) /(q+$ $\left.p e^{-a(x+L)}\right)+\theta(x) /\left(\tilde{q}+\tilde{p} e^{b(x-\widetilde{L})}\right)$. The parameter $m_{0}$ is the rest mass of Dirac particle and $m_{1}$ is a real positive and small parameter; therefore, the derivative terms including the mass $m(x)$ function are ignored in (8). So, (8) becomes

$$
\begin{aligned}
& \frac{d^{2} \Theta(x)}{d x^{2}}+\left\{[E-V(x)]^{2}-m^{2}(x)+i \frac{d V(x)}{d x}\right\} \Theta(x)=0, \\
& \frac{d^{2} \Phi(x)}{d x^{2}}+\left\{[E-V(x)]^{2}-m^{2}(x)-i \frac{d V(x)}{d x}\right\} \Phi(x)=0 .
\end{aligned}
$$

It is clearly seen from (6) and (7) that it will be sufficient to solve one of (10) and (11) to obtain the solutions describing the scattering states. Therefore, we start calculation by solving (10). Let us now define a new variable $y=-(q / p) e^{a(x+L)}$ in (10) for the region $x<0$, in this instance, (10) becomes

$$
\begin{aligned}
& a^{2} y^{2} \frac{d^{2} \Theta_{L}(y)}{d y^{2}}+a^{2} y \frac{d \Theta_{L}(y)}{d y} \\
& +\left\{\left[E+\frac{V_{0} y}{q(1-y)}\right]^{2}-m_{0}^{2}-m_{1}^{2} \frac{y^{2}}{q^{2}(1-y)^{2}}\right. \\
& \left.\quad+2 m_{0} m_{1} \frac{y}{q(1-y)}-i a y \frac{d}{d y}\left[\frac{V_{0} y}{q(1-y)}\right]\right\} \\
& \quad \times \Theta_{L}(y)=0,
\end{aligned}
$$

and getting a trial wave function $\Theta_{L}(y)=y^{\zeta}(1-y)^{\lambda} w(y)$, (12) reduces to the Gaussian differential equation [33]

$$
\begin{gathered}
y(1-y) \frac{d^{2} w(y)}{d y^{2}}+[1+2 \zeta-y(2 \zeta+2 \lambda+1)] \frac{w(y)}{d y} \\
-(\zeta+\lambda+\gamma)(\zeta+\lambda-\gamma) w(y)=0,
\end{gathered}
$$

where

$$
\begin{gathered}
\zeta=\frac{i k}{a}, \quad \lambda=\frac{1}{2}+\sqrt{\left(\frac{1}{2}+\frac{i V_{0}}{a q}\right)^{2}+\frac{m_{1}^{2}}{a^{2} q^{2}}}, \quad \gamma=\frac{i p_{1}}{a}, \\
p_{1}^{2}=\left(E-\frac{V_{0}}{q}\right)^{2}-\left(m_{0}+\frac{m_{1}}{q}\right)^{2}, \quad k=\sqrt{E^{2}-m_{0}^{2}} .
\end{gathered}
$$

The solution of (13) is found in the form of hypergeometric functions, as follows:

$$
\begin{aligned}
w(y)= & N_{12} F_{1}(\zeta+\lambda+\gamma, \zeta+\lambda-\gamma, 1+2 \zeta ; y) \\
& +N_{2} y^{-2 \zeta} \\
& \times{ }_{2} F_{1}(-\zeta+\lambda+\gamma,-\zeta+\lambda-\gamma, 1-2 \zeta ; y)
\end{aligned}
$$

so, the whole left-hand solution becomes

$$
\begin{aligned}
& \Theta_{L}(y) \\
& =N_{1} y^{\zeta}(1-y)_{2}^{\lambda} F_{1}(\zeta+\lambda+\gamma, \zeta+\lambda-\gamma, 1+2 \zeta ; y) \\
& \quad+N_{2} y^{-\zeta}(1-y)^{\lambda} \\
& \quad \times{ }_{2} F_{1}(-\zeta+\lambda+\gamma,-\zeta+\lambda-\gamma, 1-2 \zeta ; y) .
\end{aligned}
$$

Now, we can make the process to find the solution for the region $x>0$. Choosing a new variable $z=(-\widetilde{q} / \widetilde{p}) e^{-b(x-\widetilde{L})}$, (10) is written as

$$
\begin{aligned}
& b^{2} z^{2} \frac{d^{2} \Theta_{R}(z)}{d z^{2}}+b^{2} z \frac{d \Theta_{R}(z)}{d z} \\
& +\left\{\left[E+\frac{V_{0} z}{\widetilde{q}(1-z)}\right]^{2}-m_{0}^{2}-m_{1}^{2} \frac{z^{2}}{\widetilde{q}^{2}(1-z)^{2}}\right. \\
& \left.\quad+2 m_{0} m_{1} \frac{z}{\widetilde{q}(1-z)}+i b z \frac{d}{d z}\left[\frac{V_{0} z}{\widetilde{q}(1-z)}\right]\right\} \Theta_{R}(z)=0,
\end{aligned}
$$

and assuming a test wave function $\Theta_{R}(z)=z^{\tilde{\zeta}}(1-z)^{-\tilde{\lambda}} v(z)$, the solution of (17) becomes the following form:

$$
\begin{aligned}
v(z)= & N_{32} F_{1}(\widetilde{\zeta}-\tilde{\lambda}+\widetilde{\gamma}, \widetilde{\zeta}-\tilde{\lambda}-\widetilde{\gamma}, 1+2 \widetilde{\zeta} ; z) \\
& +N_{4} z^{-2 \widetilde{\zeta}}{ }_{2} F_{1}(-\widetilde{\zeta}-\tilde{\lambda}+\widetilde{\gamma},-\widetilde{\zeta}-\tilde{\lambda}-\tilde{\gamma}, 1-2 \widetilde{\zeta} ; z) .
\end{aligned}
$$


In this case, the whole right-hand solution is

$$
\begin{aligned}
\Theta_{R}(z)= & N_{3} z^{\tilde{\zeta}}(1-z)^{-\tilde{\lambda}}{ }_{2} F_{1}(\tilde{\zeta}-\tilde{\lambda}+\tilde{\gamma}, \tilde{\zeta}-\tilde{\lambda}-\tilde{\gamma}, 1+2 \widetilde{\zeta} ; z) \\
& +N_{4} z^{-\widetilde{\zeta}}(1-z)^{-\tilde{\lambda}} \\
& \times{ }_{2} F_{1}(-\tilde{\zeta}-\tilde{\lambda}+\tilde{\gamma},-\widetilde{\zeta}-\tilde{\lambda}-\tilde{\gamma}, 1-2 \widetilde{\zeta} ; z),
\end{aligned}
$$

where

$$
\begin{gathered}
\tilde{\zeta}=\frac{i k}{b}, \quad \tilde{\lambda}=-\frac{1}{2}+\sqrt{\left(\frac{1}{2}-\frac{i V_{0}}{b \widetilde{q}}\right)^{2}+\frac{m_{1}^{2}}{b^{2} \widetilde{q}^{2}}}, \quad \tilde{\gamma}=\frac{i \widetilde{p_{1}}}{b}, \\
{\widetilde{p_{1}}}^{2}=\left(E-\frac{V_{0}}{\tilde{q}}\right)^{2}-\left(m_{0}+\frac{m_{1}}{\widetilde{q}}\right)^{2}, \quad k=\sqrt{E^{2}-m_{0}^{2}} .
\end{gathered}
$$

In order to investigate the transmission $(T)$ and reflection $(R)$ coefficients, the solutions obtained above have to be used asymptotic behaviors as $x \rightarrow-\infty$ and $x \rightarrow+\infty$. If $x \rightarrow$ $-\infty$ is chosen, the left-hand solution can be written as

$$
\begin{aligned}
\Theta_{L}(x) & \longrightarrow N_{1} e^{-\pi(k / a)}\left(\frac{q}{p}\right)^{i(k / a)} e^{i k(x+L)} \\
& +N_{2} e^{\pi(k / a)}\left(\frac{q}{p}\right)^{-i(k / a)} e^{-i k(x+L)},
\end{aligned}
$$

and if $x \rightarrow+\infty$ is chosen, the right-hand solution is

$$
\Theta_{R}(x) \longrightarrow N_{4} e^{\pi(k / b)}\left(\frac{\tilde{q}}{\widetilde{p}}\right)^{-i(k / b)} e^{i k(x-\widetilde{L})} .
$$

The one-dimensional electric current density for the Dirac particle is given as the following form:

$$
J(x)=\frac{1}{2}\left[|\Theta(x)|^{2}-|\Phi(x)|^{2}\right] ;
$$

so as to find electric current density, (21) and (22) are inserted into (6). In this case, the asymptotic behaviors for $\Phi_{L}(x)$ and $\Phi_{R}(x)$ become

$$
\begin{aligned}
\Phi_{L}(x) \longrightarrow & N_{1} e^{-\pi(k / a)}\left(\frac{q}{p}\right)^{i(k / a)}\left[\frac{E-k}{m(x)}\right] e^{i k(x+L)} \\
& +N_{2} e^{\pi(k / a)}\left(\frac{q}{p}\right)^{-i(k / a)}\left[\frac{E+k}{m(x)}\right] e^{-i k(x+L)}, \\
\Phi_{R}(x) \longrightarrow & N_{4} e^{\pi(k / b)}\left(\frac{\widetilde{q}}{\widetilde{p}}\right)^{-i(k / b)}\left[\frac{E-k}{m(x)}\right] e^{i k(x-\widetilde{L})} .
\end{aligned}
$$

Using (21), (22), and (24) for electric current density given in (23), $j_{\text {inc. }}, j_{\text {trans. }}$, and $j_{\text {ref. }}$ are calculated and the reflection and transmission coefficients are found as follows, respectively:

$$
\begin{gathered}
R=\frac{j_{\text {ref. }}}{j_{\text {inc. }}}=\left|\frac{N_{2}}{N_{1}}\right|^{2}\left(\frac{E+k}{E-k}\right) e^{4 \pi k / a}, \\
T=\frac{j_{\text {trans. }}}{j_{\text {inc. }}}=\left|\frac{N_{4}}{N_{1}}\right|^{2} e^{2 \pi k(1 / a+1 / b)},
\end{gathered}
$$

TABLE 2: The abbreviations taking part in (26), (34), and (35).

$$
\begin{aligned}
& \beta=\frac{q}{p}, \widetilde{\beta}=\frac{\widetilde{q}}{\tilde{p}} \\
& B_{1}=\widetilde{\beta}^{-\widetilde{\zeta}} e^{-b \tilde{\tilde{L}} \widetilde{L}}\left(1-\widetilde{\beta} e^{b \widetilde{L}}\right)^{-\tilde{\lambda}} \\
& B_{2}=\beta^{\zeta} e^{a \zeta L}\left(1-\beta e^{a L}\right)^{\lambda} \\
& B_{3}=\beta^{-\zeta} e^{-a \zeta L}\left(1-\beta e^{a L}\right)^{\lambda} \\
& B_{4}=b \tilde{\zeta} \widetilde{\beta}^{-\widetilde{\zeta}} e^{-b \tilde{\zeta} \widetilde{L}}\left(1-\widetilde{\beta} e^{b \widetilde{L}}\right)^{-\tilde{\lambda}} \\
& B_{5}=-b \tilde{\lambda} \widetilde{\beta}^{-\widetilde{\zeta}+1} e^{-b \tilde{L}(\widetilde{\zeta}-1)}\left(1-\widetilde{\beta} e^{b \tilde{L}}\right)^{-\tilde{\lambda}-1} \\
& B_{6}=-b \widetilde{\beta}^{-\widetilde{\zeta}+1} e^{-b \tilde{L}(\widetilde{\zeta}-1)}\left(1-\widetilde{\beta} e^{b \widetilde{L}}\right)^{-\tilde{\lambda}} \frac{(-\widetilde{\zeta}-\tilde{\lambda}+\widetilde{\gamma})(-\widetilde{\zeta}-\tilde{\lambda}-\tilde{\gamma})}{1-2 \widetilde{\zeta}} \\
& B_{7}=a \zeta \beta^{\zeta} e^{a \zeta L}\left(1-\beta e^{a L}\right)^{\lambda} \\
& B_{8}=-a \lambda \beta^{\zeta+1} e^{a L(\zeta+1)}\left(1-\beta e^{a L}\right)^{\lambda-1} \\
& B_{9}=a \beta^{\zeta+1} e^{a L(\zeta+1)}\left(1-\beta e^{a L}\right)^{\lambda} \frac{(\zeta+\lambda+\gamma)(\zeta+\lambda-\gamma)}{1+2 \zeta} \\
& B_{10}=-a \zeta \beta^{-\zeta} e^{-a \zeta L}\left(1-\beta e^{a L}\right)^{\lambda} \\
& B_{11}=-a \lambda \beta^{-\zeta+1} e^{-a L(\zeta-1)}\left(1-\beta e^{a L}\right)^{\lambda-1} \\
& B_{12}=a \beta^{-\zeta+1} e^{-a L(\zeta-1)}\left(1-\beta e^{a L}\right)^{\lambda} \frac{(-\zeta+\nu+\gamma)(-\zeta+\lambda-\gamma)}{1-2 \zeta} \\
& F_{1}={ }_{2} F_{1}\left(-\widetilde{\zeta}-\tilde{\lambda}+\tilde{\gamma},-\widetilde{\zeta}-\tilde{\lambda}-\tilde{\gamma}, 1-2 \widetilde{\zeta} ; \widetilde{\beta} e^{b \widetilde{L}}\right) \\
& F_{2}={ }_{2} F_{1}\left(\zeta+\lambda+\gamma, \zeta+\lambda-\gamma, 1+2 \zeta ; \beta e^{a L}\right) \\
& F_{3}={ }_{2} F_{1}\left(-\zeta+\lambda+\gamma,-\zeta+\lambda-\gamma, 1-2 \zeta ; \beta e^{a L}\right) \\
& F_{4}={ }_{2} F_{1}\left(-\widetilde{\zeta}-\tilde{\lambda}+\widetilde{\gamma}+1,-\widetilde{\zeta}-\widetilde{\lambda}-\tilde{\gamma}+1,2-2 \widetilde{\zeta} ; \widetilde{\beta} e^{b \widetilde{L}}\right) \\
& F_{5}={ }_{2} F_{1}\left(\zeta+\lambda+\gamma+1, \zeta+\lambda-\gamma+1,2+2 \zeta ; \beta e^{a L}\right) \\
& F_{6}={ }_{2} F_{1}\left(-\zeta+\lambda+\gamma+1,-\zeta+\lambda-\gamma+1,2-2 \zeta ; \beta e^{a L}\right) \\
& \widetilde{F}_{1}={ }_{2} F_{1}\left(-\widetilde{\lambda}+\widetilde{\gamma_{c}},-\widetilde{\lambda}-\widetilde{\gamma_{c}}, 1 ; \widetilde{\beta} e^{b \widetilde{L}}\right) \\
& \widetilde{F}_{2}={ }_{2} F_{1}\left(\lambda+\gamma_{c}, \lambda-\gamma_{c}, 1 ; \beta e^{a L}\right) \\
& \widetilde{F}_{4}={ }_{2} F_{1}\left(-\widetilde{\lambda}+\widetilde{\gamma_{c}}+1,-\widetilde{\lambda}-\widetilde{\gamma}_{c}+1,2 ; \widetilde{\beta} e^{b \widetilde{L}}\right) \\
& \underline{\tilde{F}_{5}}={ }_{2} F_{1}\left(\lambda+\gamma_{c}+1, \lambda-\gamma_{c}+1,2 ; \beta e^{a L}\right)
\end{aligned}
$$

where $j_{\text {inc. }}, j_{\text {trans. }}$, and $j_{\text {ref. }}$ represent the incident, transmitted, and reflected currents, respectively. Finally, to investigate numerically the reflection and transmission coefficients, the continuity condition and the first derivative of the wave function are used. So, $N_{2} / N_{1}$ and $N_{4} / N_{1}$, taking part in the above expressions, are found as

$$
\begin{aligned}
\frac{N_{2}}{N_{1}}=( & {\left[\left(B_{4}+B_{5}\right) F_{1}+B_{6} F_{4}\right] B_{2} F_{2} } \\
& \left.-\left[\left(B_{7}+B_{8}\right) F_{2}+B_{9} F_{5}\right] B_{1} F_{1}\right) \\
\times & \left(\left[\left(B_{10}+B_{11}\right) F_{3}+B_{12} F_{6}\right] B_{1} F_{1}\right. \\
& \left.-\left[\left(B_{4}+B_{5}\right) F_{1}+B_{6} F_{4}\right] B_{3} F_{3}\right)^{-1}, \\
\frac{N_{4}}{N_{1}}=( & \left.\left(B_{7}+B_{8}\right) F_{2}+B_{9} F_{5}\right] B_{3} F_{3} \\
& \left.-\left[\left(B_{10}+B_{11}\right) F_{3}+B_{12} F_{6}\right] B_{2} F_{2}\right) \\
\times & \left(\left[\left(B_{4}+B_{5}\right) F_{1}+B_{6} F_{4}\right] B_{3} F_{3}\right. \\
& \left.-\left[\left(B_{10}+B_{11}\right) F_{3}+B_{12} F_{6}\right] B_{1} F_{1}\right)^{-1},
\end{aligned}
$$

where the constants are given in Table 2. 


\section{The Equation of Energy Eigenvalues}

The purpose of this section is to derive an equation that is known as the bound state condition for the energy eigenvalues. For this, $-V_{0}$ is put instead of $V_{0}$ in the potential given in (1) and the new variable is defined as variable $y=$ $-(q / p) e^{a(x+L)}$ in the region $x<0$. As a result, (10) is written in the following form:

$$
\begin{aligned}
& a^{2} y^{2} \frac{d^{2} \Theta_{L}(y)}{d y^{2}}+a^{2} y \frac{d \Theta_{L}(y)}{d y} \\
& +\left\{\left[E-\frac{V_{0} y}{q(1-y)}\right]^{2}-m_{0}^{2}-m_{1}^{2} \frac{y^{2}}{q^{2}(1-y)^{2}}\right. \\
& \left.\quad+2 m_{0} m_{1} \frac{y}{q(1-y)}+i a y \frac{d}{d y}\left[\frac{V_{0} y}{q(1-y)}\right]\right\} \\
& \quad \times \Theta_{L}(y)=0 .
\end{aligned}
$$

Taking a wave function as $\Theta_{L}(y)=y^{\zeta_{1}}(1-y)^{\lambda_{1}} h(y)$, the solution of (27) is obtained as

$$
\begin{aligned}
\Theta_{L}(y) & \\
= & A_{1} y^{\zeta}(1-y)^{\lambda_{1}} \\
& \times{ }_{2} F_{1}\left(\zeta_{1}+\lambda_{1}+\gamma_{1}, \zeta_{1}+\lambda_{1}-\gamma_{1}, 1+2 \zeta_{1} ; y\right) \\
& +A_{2} y^{-\zeta_{1}}(1-y)^{\lambda_{1}} \\
& \times{ }_{2} F_{1}\left(-\zeta_{1}+\lambda_{1}+\gamma_{1},-\zeta_{1}+\lambda_{1}-\gamma_{1}, 1-2 \zeta_{1} ; y\right),
\end{aligned}
$$

where

$$
\begin{array}{rlrl}
\zeta_{1}=\frac{k_{1}}{a}, & \lambda_{1} & =\frac{1}{2}+\sqrt{\left(\frac{1}{2}-\frac{i V_{0}}{a q}\right)^{2}+\frac{m_{1}^{2}}{a^{2} q^{2}}} \\
\gamma_{1}=\frac{i p_{2}}{a}, & p_{2}^{2} & =\left(E+\frac{V_{0}}{q}\right)^{2}-\left(m_{0}+\frac{m_{1}}{q}\right)^{2}, \\
k_{1} & =\sqrt{m_{0}^{2}-E^{2}} .
\end{array}
$$

Similarly, choosing the variable $z=(-\widetilde{q} / \widetilde{p}) e^{-b(x-\widetilde{L})}$ and wave function $\Theta_{R}(z)=z^{\widetilde{\zeta_{1}}}(1-z)^{-\widetilde{\lambda_{1}}} f(z)$ in the region $x>0$, the positive region solution is found as

$$
\begin{aligned}
\Theta_{R}(z)= & A_{3} z^{\widetilde{\zeta_{1}}}(1-z)^{-\widetilde{\lambda_{1}}} \\
& \times{ }_{2} F_{1}\left(\widetilde{\zeta_{1}}-\widetilde{\lambda_{1}}+\widetilde{\gamma_{1}}, \widetilde{\zeta_{1}}-\widetilde{\lambda_{1}}-\widetilde{\gamma_{1}}, 1+2 \widetilde{\zeta_{1}} ; z\right) \\
& +A_{4} z^{-\widetilde{\zeta_{1}}}(1-z)^{-\widetilde{\lambda_{1}}} \\
& \times{ }_{2} F_{1}\left(-\widetilde{\zeta_{1}}-\widetilde{\lambda_{1}}+\widetilde{\gamma_{1}},-\widetilde{\zeta_{1}}-\widetilde{\lambda_{1}}-\widetilde{\gamma_{1}}, 1-2 \widetilde{\zeta_{1}} ; z\right),
\end{aligned}
$$

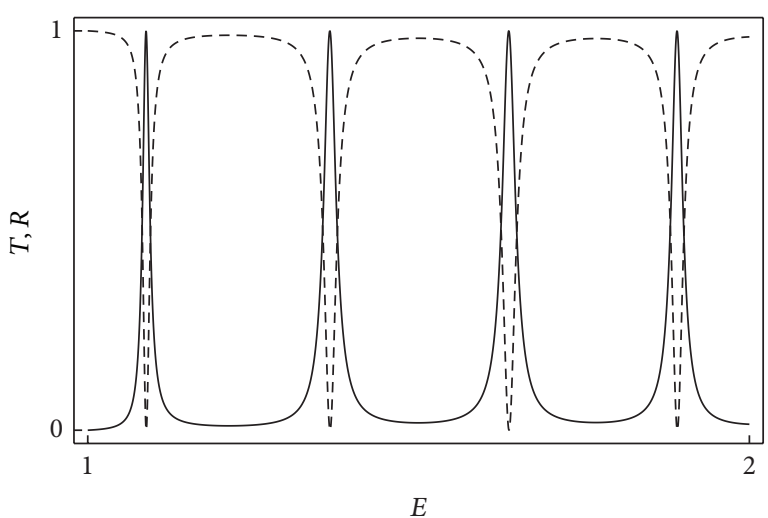

FIgUre 2: The plot of unitarity condition, $R+T=1$, with the position dependent mass for $q=1.5, \widetilde{q}=1.5, p=2, \widetilde{p}=2, L=5, \widetilde{L}=5$, $a=3, b=3, V_{0}=5, m_{0}=1$, and $m_{1} \rightarrow 0$.

where

$$
\begin{array}{rlrl}
\widetilde{\zeta_{1}}=\frac{k_{1}}{b}, & \widetilde{\lambda_{1}} & =-\frac{1}{2}+\sqrt{\left(\frac{1}{2}+\frac{i V_{0}}{b \widetilde{q}}\right)^{2}+\frac{m_{1}^{2}}{b^{2} \widetilde{q}^{2}},} \\
\widetilde{\gamma_{1}}=\frac{i \widetilde{p_{2}}}{b}, & {\widetilde{p_{2}}}^{2} & =\left(E+\frac{V_{0}}{\widetilde{q}}\right)^{2}-\left(m_{0}+\frac{m_{1}}{\widetilde{q}}\right)^{2}, \\
k_{1} & =\sqrt{m_{0}^{2}-E^{2}} .
\end{array}
$$

Because of the boundary conditions (which mean the wave functions go to zero at infinity), the wave functions given in (28) and (30) are written as

$$
\begin{aligned}
\Theta_{L}(y)= & A_{1} y^{\zeta}(1-y)^{\lambda_{1}} \\
& \times{ }_{2} F_{1}\left(\zeta_{1}+\lambda_{1}+\gamma_{1}, \zeta_{1}+\lambda_{1}-\gamma_{1}, 1+2 \zeta_{1} ; y\right), \\
\Theta_{R}(z)= & A_{3} z^{\widetilde{\zeta_{1}}}(1-z)^{-\widetilde{\lambda_{1}}} \\
& \times{ }_{2} F_{1}\left(\widetilde{\zeta_{1}}-\widetilde{\lambda_{1}}+\widetilde{\gamma_{1}}, \widetilde{\zeta_{1}}-\widetilde{\lambda_{1}}-\widetilde{\gamma_{1}}, 1+2 \widetilde{\zeta_{1}} ; z\right) .
\end{aligned}
$$

In order to find an equation for energy eigenvalues, the continuity conditions of the wave function are used, $\Theta_{L}(x=$ $0)=\Theta_{R}(x=0)$ and $d \Theta_{L} /\left.d x\right|_{x=0}=d \Theta_{R} /\left.d x\right|_{x=0}$. After performing the necessary calculations, an equation for energy eigenvalues is obtained as follows:

$$
\begin{aligned}
& {\left[\left(D_{3}+D_{4}\right) F_{7}+D_{5} F_{9}\right] D_{2} F_{8}} \\
& \quad-\left[\left(D_{6}+D_{7}\right) F_{8}+D_{8} F_{10}\right] D_{1} F_{7}=0,
\end{aligned}
$$

where the constants are given Table 3 .

\section{Results and Discussions}

4.1. Interpretation of the Drawings and Comparison of the Results with Previous Ones. In Figure 2, while the parameter 
TABLE 3: The constants given in (33) and (37).

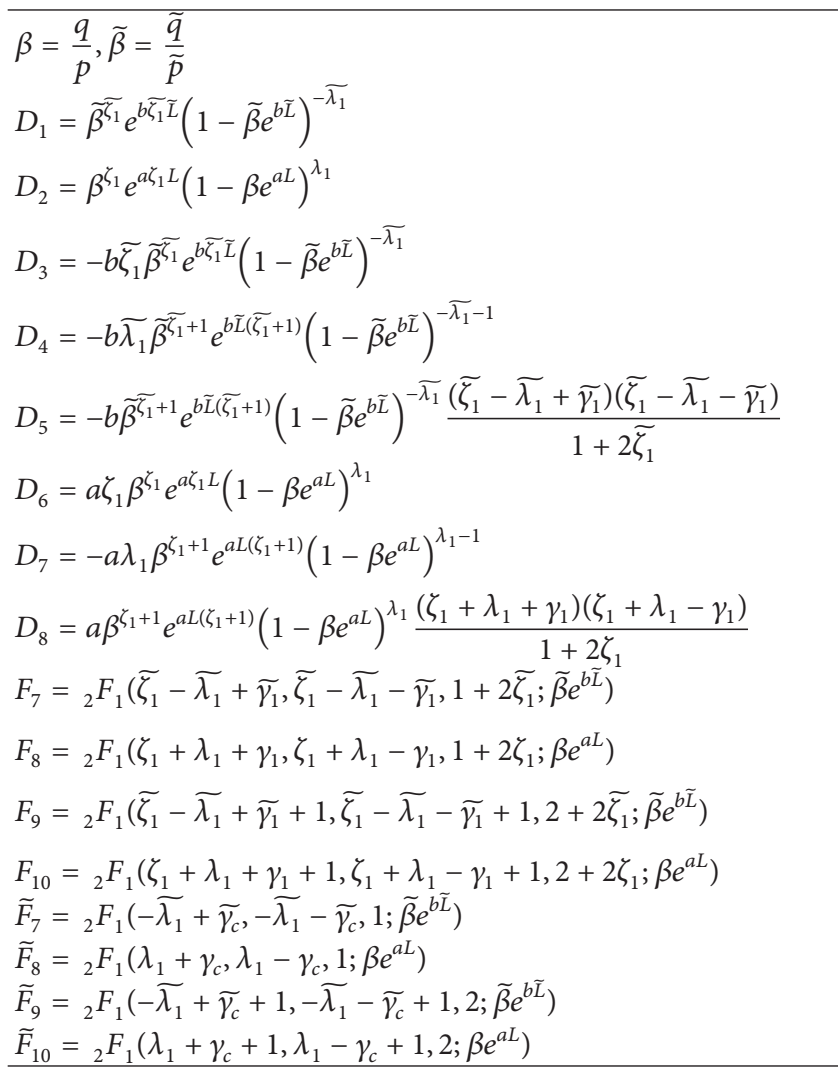

$m_{1}$ goes to zero and the GAWSp is a symmetric potential, one can clearly see that the unitary condition, $R+T=1$, is provided in the GAWSp for the position dependent mass Dirac particle. On the other hand, if the parameter $m_{1}$ does not go to zero and the GAWSp is an asymmetric one, the unitary condition is not provided. Figure 3 shows the effect of position dependent mass for the Dirac particle within the GAWSp on the transmission coefficient. In this figure, it is seen that the presence of variable mass causes the formation of a wider line according to the previous graph that is one of the constant masses. One of the advantages of this work is that, by using the GAWSp solutions for the Dirac particle with variable mass, the transmission and reflection coefficients are directly obtained without doing calculation for the potentials which are given in Table 1. For example, in the numerical results of the GAWSp, if one chooses $a=b, L=\widetilde{L}, p=\widetilde{p}=1$, and $q=\widetilde{q}=1$, one can acquire the scattering states of the Woods-Saxon potential, by putting $a=b, L=\widetilde{L}=0$, $p=\tilde{p}=1, \tilde{q} \rightarrow-q$, and $q \rightarrow-q$, the scattering states of the Hulthen potential are obtained, substituting $a=b, L=\widetilde{L}$, $p=\widetilde{p}=1$, and $q=\widetilde{q}=0$, the scattering states of the cusp potential are found. Figures 4-6 are drawn using the GAWSp solutions for the Dirac particle. Figure 4 displays a plot of the transmission coefficient describing the behavior of the Dirac particle in the Woods-Saxon potential versus the energy and the potential strength for both the position dependent mass and the constant mass. From Figure 4, it is seen, in the presence of position dependent mass, that the zero region of the transmission coefficient expands. At the same time, the results of Figure 4 are compatible with [7,20]. Figure 5 shows the effect of the position dependent Dirac particle for the Hulthen potential on the transmission coefficient. The data drawn from Figure 5 prove that the values of the transmission coefficient in the case of position dependent mass decrease. Furthermore, these results are similar to [12, 22]. Figure 6 displays the effect of the Dirac particle for the cusp potential on the transmission coefficient. Figure 7 shows a plot of the transmission coefficients obtained for the Dirac particle with the position dependent mass against the energy and the potential strength for the GAWS, the Woods-Saxon, the Hulthen, and the cusp potentials. In the left panel which is located in this figure, while the transmission resonance appears for the GAWS and Woods-Saxon potentials at lower values of energy, it does not exist for the Hulthen and the cusp potentials at same values of energy. In the right panel taking part in the same figure, while the transmission resonance peaks are more for the Woods-Saxon potential at higher values of potential strength $\left(V_{0}\right)$, the transmission resonance does not occur for the cusp potential at same values of $V_{0}$. Moreover, if one wants to achieve the scattering solutions of the asymmetric Hulthen and the asymmetric cusp potentials, one can use the solutions describing the scattering states, of the GAWSp, found in this study.

In addition, by using (33), energy eigenvalues can be calculated numerically. As the bound states are considered, $k_{1}$ must be real which means $E<m_{0}$ in (29) and (31) and also values of energy must be $-|V(x)|<E$. Besides, the energy takes the values providing the two conditions that $-\left|V_{0} /\left(q+p e^{-a L}\right)\right|<E<m_{0}$ in the region $x<0$ and $-\left|V_{0} /\left(\widetilde{q}+\widetilde{p} e^{-b \widetilde{L}}\right)\right|<E<m_{0}$ in the region $x>0$ due to the asymmetric form of the GAWS well. As the scattering states are handled, by using the GAWS well solutions for the Dirac particle, the numerical energy values are directly obtained without doing calculation for the asymmetric Hulthen and asymmetric cusp wells. Additionally, the energy takes the values providing these conditions that $-\left|V_{0} /(1-q)\right|<E<m_{0}$ in the region $x<0$ and $-\left|V_{0} /(1-\widetilde{q})\right|<E<m_{0}$ in the region $x>0$ for the asymmetric Hulthen well and $-\left|V_{0}\right|<E<m_{0}$ in the regions $x<0$ and $x>0$ for the asymmetric cusp well. The numerical energy eigenvalues containing all cases which are given above for asymmetric potentials are found in Table 4 .

\subsection{Conditions for Transmission Resonance and Supercritical-} ity. The aim of this section is to obtain the conditions describing a transmission resonance (providing the requirement that the transmission coefficient is unity) and supercriticality (while the bound state of the Dirac particle is at $E=-m_{0}$ ).

To find a condition describing the transmission resonance for the scattering states, $N_{2} / N_{1}$ taking part in (26), must be equal to zero. From this situation, the following expression is attained:

$$
\begin{aligned}
& {\left[\left(B_{4}+B_{5}\right) F_{1}+B_{6} F_{4}\right] B_{2} F_{2}} \\
& \quad-\left[\left(B_{7}+B_{8}\right) F_{2}+B_{9} F_{5}\right] B_{1} F_{1}=0,
\end{aligned}
$$




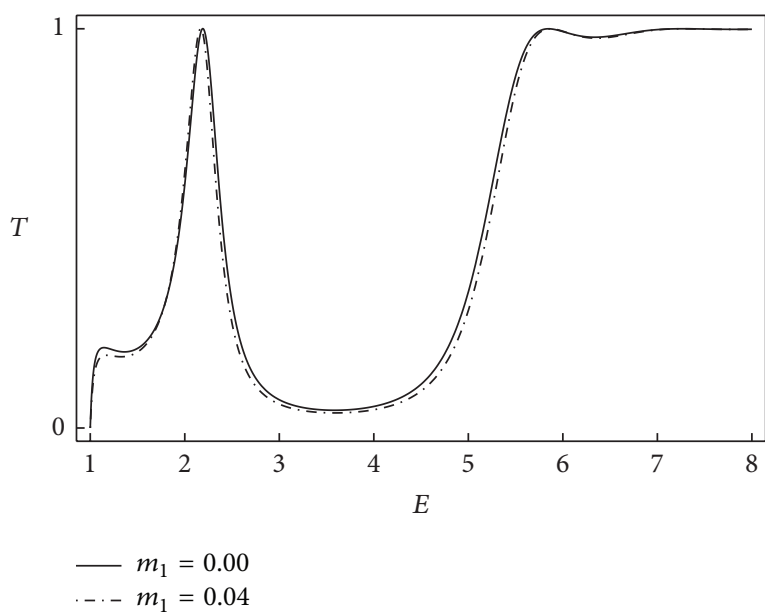

(a)

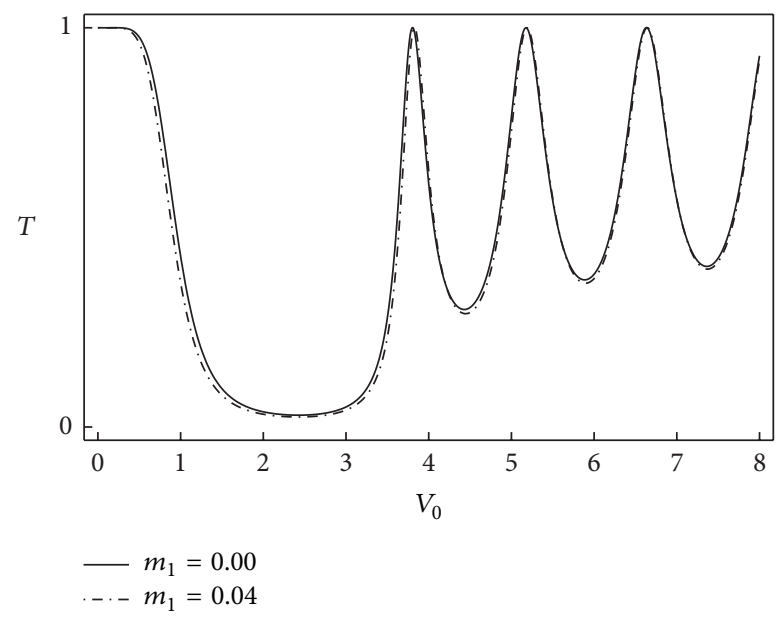

(b)

FIGURE 3: The effect of position dependent mass for the GAWSp on transmission coefficient. (a) shows $V_{0}=4, L=1.2, \widetilde{L}=1.1, a=10$, $b=10, p=2.2, \widetilde{p}=2.1, q=1, \widetilde{q}=1$, and $m_{0}=1$. (b) shows $E=2, L=1.2, \widetilde{L}=1.1, a=10, b=10, p=2.2, \widetilde{p}=2.1, q=1, \widetilde{q}=1$, and $m_{0}=1$.

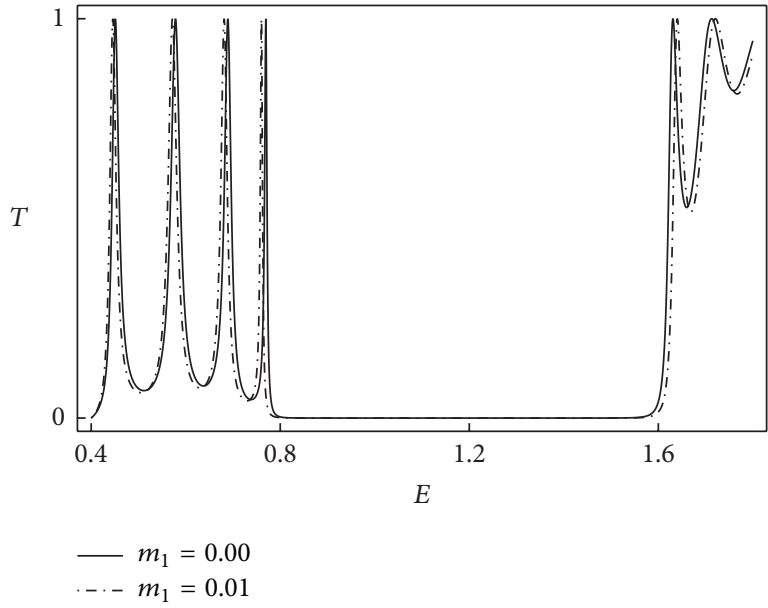

(a)

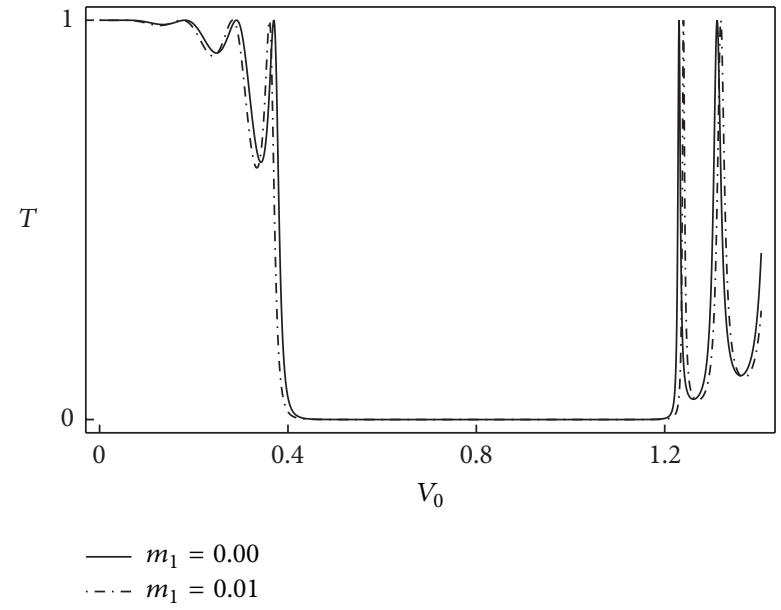

(b)

FIgURE 4: The effect of position dependent mass for the Woods-Saxon potential on transmission coefficient. (a) shows $V_{0}=1.2, L=10$, $\widetilde{L}=10, a=3, b=3, p=1, \widetilde{p}=1, q=1, \widetilde{q}=1$, and $m_{0}=0.4$. (b) shows $E=0.8, L=10, \widetilde{L}=10, a=3, b=3, p=1, \widetilde{p}=1, q=1, \widetilde{q}=1$, and $m_{0}=0.4$.

where the constants are given in Table 2. The above equation takes the following form in the limit of low momentum $(E \rightarrow$ $m_{0}$ which leads to $\zeta=\widetilde{\zeta}=0$ ):

$$
\begin{aligned}
& {\left[(-\widetilde{\lambda} b) \widetilde{\beta} e^{b \widetilde{L}}\left(1-\widetilde{\beta} e^{b \widetilde{L}}\right)^{-\widetilde{\lambda}-1} \widetilde{F_{1}}\right.} \\
& \left.\quad-b \widetilde{\beta} e^{b \widetilde{L}}\left(1-\widetilde{\beta} e^{b \widetilde{L}}\right)^{-\widetilde{\lambda}}\left(-\widetilde{\lambda}+\widetilde{\gamma_{c}}\right)\left(-\widetilde{\lambda}-\widetilde{\gamma_{c}}\right) \widetilde{F_{4}}\right] \\
& \quad \times\left(1-\beta e^{a L}\right)^{\lambda} \widetilde{F_{2}} \\
& \quad-\left[(-a \lambda) \beta e^{a L}\left(1-\beta e^{a L}\right)^{\lambda-1} \widetilde{F_{2}}\right.
\end{aligned}
$$

$$
\begin{aligned}
& \left.+a \beta e^{a L}\left(1-\beta e^{a L}\right)^{\lambda}\left(\lambda+\gamma_{c}\right)\left(\lambda-\gamma_{c}\right) \widetilde{F_{5}}\right] \\
& \times\left(1-\widetilde{\beta} e^{b \widetilde{L}}\right)^{-\widetilde{\lambda}} \widetilde{F_{1}}=0,
\end{aligned}
$$

where

$$
\begin{array}{ll}
\gamma_{c}=\frac{i p_{c}}{a}, & p_{c}^{2}=\frac{V_{0}^{2}-m_{1}^{2}}{q^{2}}-2 m_{0} \frac{V_{0}+m_{1}}{q}, \\
\widetilde{\gamma}_{c}=\frac{i \widetilde{p_{c}}}{b}, & \widetilde{p}_{c}^{2}=\frac{V_{0}^{2}-m_{1}^{2}}{\widetilde{q}^{2}}-2 m_{0} \frac{V_{0}+m_{1}}{\tilde{q}} .
\end{array}
$$

The abbreviations taking part in (35) are given in Table 2. 


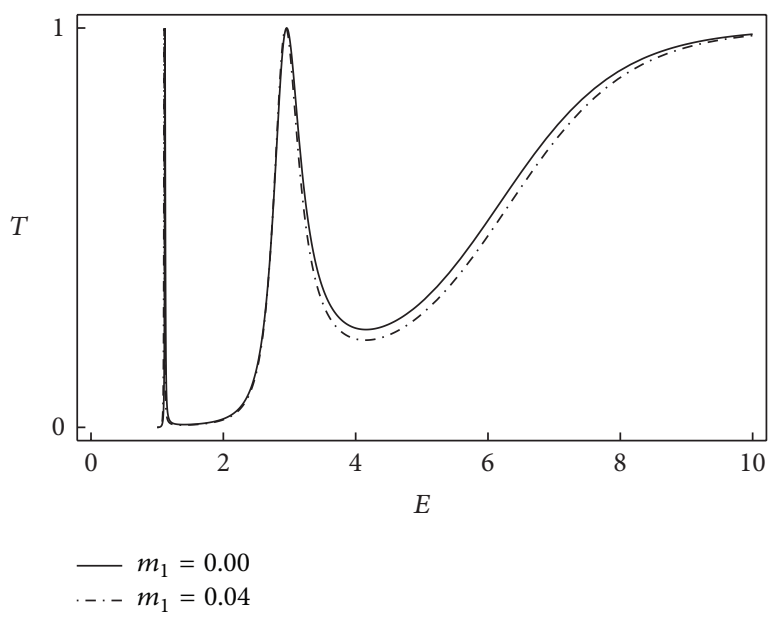

(a)

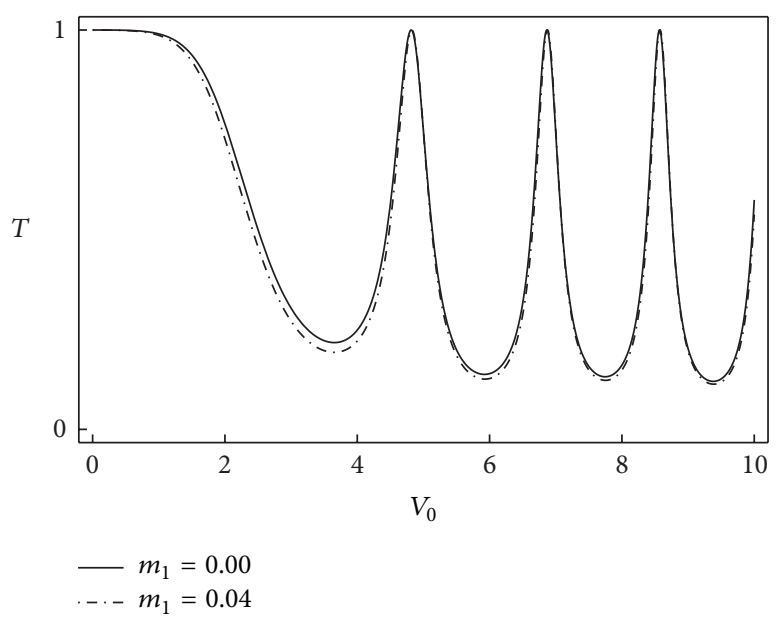

(b)

FIGURE 5: The effect of position dependent mass for the Hulthen potential on transmission coefficient. (a) shows $V_{0}=4, L=0, \widetilde{L}=0, a=1$, $b=1, p=1, \widetilde{p}=1, q=0.5, \widetilde{q}=0.5$, and $m_{0}=1$. (b) shows $E=2, L=0, \widetilde{L}=0, a=1, b=1, p=1, \widetilde{p}=1, q=0.5, \widetilde{q}=0.5$, and $m_{0}=1$.

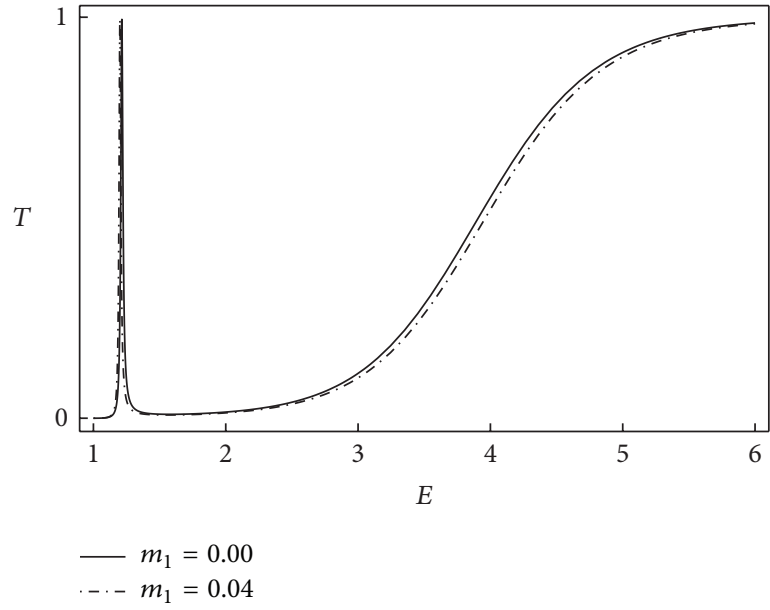

(a)

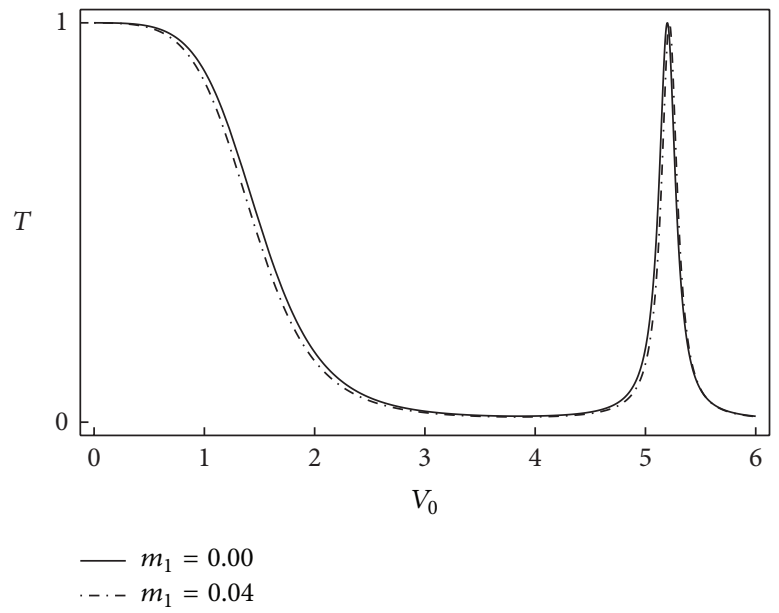

(b)

Figure 6: The effect of position dependent mass for the cusp potential on transmission coefficient. (a) shows $V_{0}=4, L=0, \widetilde{L}=0, a=1$, $b=1, p=1, \widetilde{p}=1, q=\widetilde{q} \rightarrow 0$, and $m_{0}=1$. (b) shows $E=2, L=0, \widetilde{L}=0, a=1, b=1, p=1, \widetilde{p}=1, q=\widetilde{q} \rightarrow 0$, and $m_{0}=1$.

To achieve the supercritical condition for the bound states, the low-momentum limit $\left(E \rightarrow-m_{0}\right.$ which leads to $\zeta_{1}=\widetilde{\zeta}_{1}=0$ ) is used. In this case, (33) giving the energy eigenvalues reduces to the following equation:

$$
\begin{aligned}
& {\left[\left(-\widetilde{\lambda_{1}} b\right) \widetilde{\beta} e^{b \widetilde{L}}\left(1-\widetilde{\beta} e^{b \widetilde{L}}\right)^{-\widetilde{\lambda_{1}}-1} \widetilde{F_{7}}\right.} \\
& \left.\quad-b \widetilde{\beta} e^{b \widetilde{L}}\left(1-\widetilde{\beta} e^{b \widetilde{L}}\right)^{-\widetilde{\lambda_{1}}}\left(-\widetilde{\lambda_{1}}+\widetilde{\gamma_{c}}\right)\left(-\widetilde{\lambda_{1}}-\widetilde{\gamma_{c}}\right) \widetilde{F_{9}}\right] \\
& \quad \times\left[\left(1-\beta e^{a L}\right)^{\lambda_{1}} \widetilde{F_{8}}\right] \\
& \quad-\left[\left(-a \lambda_{1}\right) \sigma e^{a L}\left(1-\beta e^{a L}\right)^{\lambda_{1}-1} \widetilde{F_{8}}\right.
\end{aligned}
$$

$$
\begin{aligned}
& \left.+a \beta e^{a L}\left(1-\beta e^{a L}\right)^{\lambda_{1}}\left(\lambda_{1}+\gamma_{c}\right)\left(\lambda_{1}-\gamma_{c}\right) \widetilde{F_{10}}\right] \\
& \times\left[\left(1-\widetilde{\beta} e^{b \widetilde{L}}\right)^{-\widetilde{\lambda_{1}} \widetilde{F_{7}}}\right]=0,
\end{aligned}
$$

where

$$
\begin{aligned}
& \gamma_{c}=\frac{i p_{c}}{a}, \quad p_{c}^{2}=\frac{V_{0}^{2}-m_{1}^{2}}{q^{2}}-2 m_{0} \frac{V_{0}+m_{1}}{q}, \\
& \widetilde{\gamma}_{c}=\frac{i \widetilde{p}_{c}}{b}, \quad \widetilde{p}_{c}^{2}=\frac{V_{0}^{2}-m_{1}^{2}}{\widetilde{q}^{2}}-2 m_{0} \frac{V_{0}+m_{1}}{\widetilde{q}} . \\
& \widetilde{F_{7}}, \widetilde{F_{8}}, \widetilde{F_{9}} \text {, and } \widetilde{F_{10}} \text { in }(37) \text { are given in Table } 3 .
\end{aligned}
$$


TABLE 4: The numerical energy values (in atomic units: $\mathrm{fm}^{-1}$ ) for asymmetric wells.

\begin{tabular}{|c|c|c|c|}
\hline \multirow{2}{*}{ The potentials } & \multirow{2}{*}{ The parameters } & \multicolumn{2}{|c|}{ The energy values } \\
\hline & & Constant mass & Effective mass \\
\hline \multirow{5}{*}{ The GAWS well } & & $m_{1}=0$ & $m_{1}=0.01$ \\
\hline & $L=2, \widetilde{L}=2.1, p=\widetilde{p}=1$ & $E_{1}=-1.95451$ & $E_{1}=-1.95857$ \\
\hline & $q=1, \widetilde{q}=1, a=0.7, b=0.8$ & $E_{2}=-0.90736$ & $E_{2}=-0.90850$ \\
\hline & $m_{0}=1, V_{0}=-3$ & $E_{3}=-0.48636$ & $E_{3}=-0.45488$ \\
\hline & & $E_{4}=0.39688$ & $E_{4}=0.39469$ \\
\hline \multirow{5}{*}{ The asymmetric Hulthen well } & $L=\widetilde{L}=0, p=\widetilde{p}=1$ & $m_{1}=0$ & $m_{1}=0.01$ \\
\hline & $q \rightarrow-q=0.5, \tilde{q} \rightarrow-q=0.5$ & $E_{1}=-1.57620$ & $E_{1}=-1.58087$ \\
\hline & $a=0.7, b=0.8$ & $E_{2}=-0.99731$ & $E_{2}=-0.99705$ \\
\hline & $m_{0}=1, V_{0}=-3$ & $E_{3}=-0.86892$ & $E_{3}=-0.86806$ \\
\hline & & $E_{4}=0.36086$ & $E_{4}=0.37312$ \\
\hline \multirow{5}{*}{ The asymmetric cusp well } & & $m_{1}=0$ & $m_{1}=0.01$ \\
\hline & $L=\widetilde{L}=0, p=\widetilde{p}=1$ & $E_{1}=-1.48765$ & $E_{1}=-1.48744$ \\
\hline & $q=\tilde{q} \rightarrow 0, a=0.7, b=0.8$ & $E_{2}=-0.98242$ & $E_{2}=-0.98242$ \\
\hline & $m_{0}=1, V_{0}=-3$ & $E_{3}=-0.35901$ & $E_{3}=-0.35901$ \\
\hline & & $E_{4}=0.35901$ & $E_{4}=0.35901$ \\
\hline
\end{tabular}

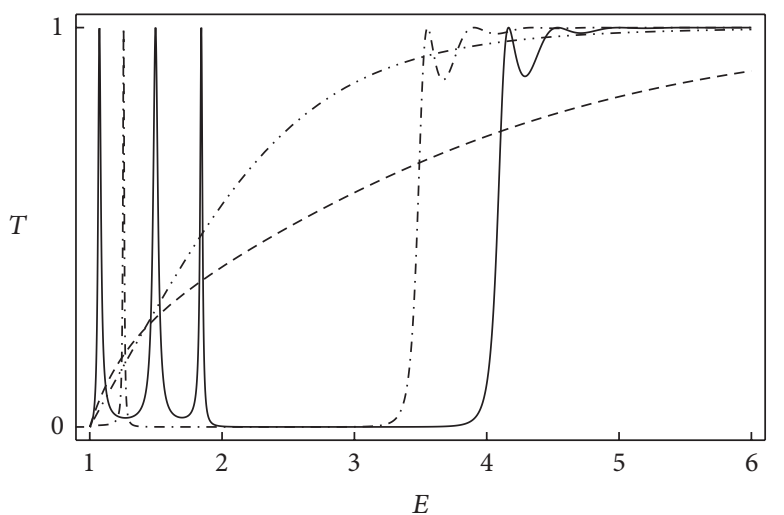

(a)

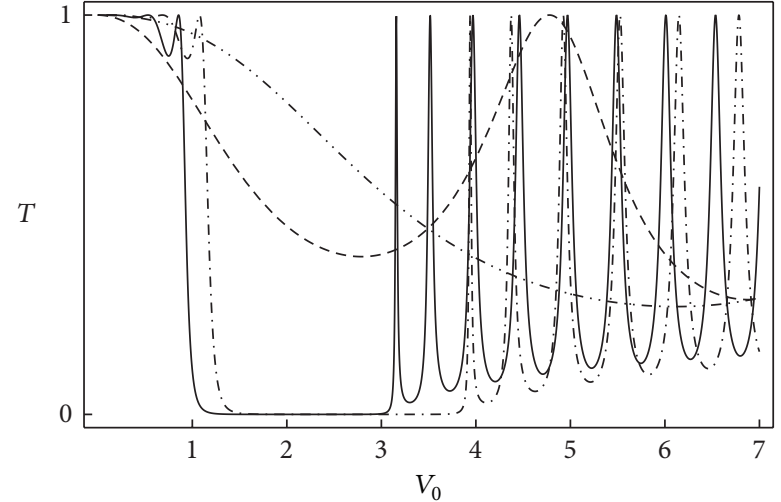

(b)

FIGURE 7: The effect of the potentials for position dependent mass on the transmission coefficient: the GAWSp for $q=\tilde{q}=1.25, a=b=4$, $L=\widetilde{L}=3, p=\widetilde{p}=1, m_{0}=1$, and $m_{1}=0.01$ (dot-dashed lines), the Woods-Saxon potential for $q=\widetilde{q}=1, L=\widetilde{L}=3, p=\widetilde{p}=1, m_{0}=1$, and $m_{1}=0.01$ (solid lines), the cusp potential for $q=\widetilde{q} \rightarrow 0, a=b=4, L=\widetilde{L}=0, p=\widetilde{p}=1, m_{0}=1$, and $m_{1}=0.01$ (dash-double-dotted lines), and the Hulthen potential for $q=\widetilde{q}=0.75, a=b=4, L=\widetilde{L}=0, p=\widetilde{p}=1, m_{0}=1$, and $m_{1}=0.01$ (dashed lines). In (a) and (b), parameters are $V_{0}=3$ and $E=2$, respectively.

When (35) and (37) are compared, it is clearly seen that these two equations are equal to each other to be $\widetilde{\lambda}=\widetilde{\lambda_{1}}$ and $\lambda=\lambda_{1}$. This case means that the GAWSp promotes a state which has the zero momentum. At the same time, this state is defined as the half-bound one.

\section{Conclusions}

The effective mass Dirac equation written for the GAWSp is solved in the approach that the parameter $m_{1}$ has very small values (as $m_{1} \rightarrow 0$ ). The transmission and reflection coefficients are acquired by utilizing the asymptotic behaviors and the boundary conditions of the obtained wave functions. Besides, the scattering states are discussed in both the effective mass and the constant mass and the scattering states for the Woods-Saxon, the Hulthen, and the cusp potentials are obtained by using the scattering solutions of the GAWSp. The energy eigenvalues found for the GAWS, the asymmetric Hulthen, and the asymmetric cusp wells are calculated numerically by using the regular wave functions. Finally, the transmission resonance condition and the equation for the supercritical state are acquired. For all that, the results obtained in this study are compared with the ones found in earlier studies and are seen to be compatible. Once and for all, when the parameter $m_{1}$ does not go to zero and the GAWSp is an asymmetric potential, the unitary condition is not provided. This result is one of the most important results of this study. This is a problem which is questionable. 


\section{Conflict of Interests}

The authors declare that there is no conflict of interests regarding the publication of this paper.

\section{Acknowledgments}

The authors wish to thank Assistant Professor Oktay Aydoğdu for many useful discussions that provided improvements to the present paper. Also, this research was partially supported by the Scientific and Technological Research Council of Turkey. This work was supported by Mersin University Scientific Research Unit (BAP-FBE F (SA) 2011-1 YL).

\section{References}

[1] L. D. Landau and E. M. Lifshitz, Quantum Mechanics, NonRelativistic Theory, Pergamon, New York, NY, USA, 3rd edition, 1977.

[2] R. G. Newton, Scattering Theory of Waves and Particles, Springer, New York, NY, USA, 2nd edition, 1982.

[3] S. Flügge, Practical Quantum Mechanics, Springer, 2nd edition, 1994.

[4] D. Bohm, Quantum Mechanics, Prentice-Hall, New York, NY, USA, 1951.

[5] N. Domhey, P. Kennedy, and A. Calogeracos, "Supercriticality and transmission resonances in the Dirac equation," Physical Review Letters, vol. 85, no. 9, pp. 1787-1790, 2000.

[6] P. Kennedy and N. Dombey, "Low momentum scattering in the Dirac equation," Journal of Physics A: Mathematical and General, vol. 35, no. 31, pp. 6645-6657, 2002.

[7] P. Kennedy, "The Woods-Saxon potential in the Dirac equation," Journal of Physics A: Mathematical and General, vol. 35, no. 3, pp. 689-698, 2002.

[8] V. M. Villalba and W. Greiner, "Scattering of a relativistic scalar particle by a cusp potential," Physical Review A, vol. 67, Article ID 052707, 2003.

[9] C. Rojas and V. M. Villalba, "Scattering of vector bosons by a n asymmetric Hulthen potential," Physical Review A, vol. 71, Article ID 052101, 2005.

[10] J. Guo and X. Fang, "Scattering of a Klein-Gordon particle by a Hulthén potential," Canadian Journal of Physics, vol. 87, no. 9, pp. 1021-1024, 2009.

[11] V. M. Villalba and C. Rojas, "Scattering of a relativistic scalar particle by a cusp potential," Physics Letters A, vol. 362, no. 1, pp. 21-25, 2007.

[12] J. Y. Guo, Y. Yu, and S. W. Jin, "Transmission resonance for a Dirac particle in a one-dimensional Hulthén potential," Central European Journal of Physics, vol. 7, no. 1, pp. 168-174, 2009.

[13] K. Sogut and A. Havare, "Transmission resonances in the Duffin-Kemmer-Petiau equation in $(1+1)$ dimensions for an asymmetric cusp potential," Physica Scripta, vol. 82, no. 4, Article ID 045013, 2010.

[14] K. Sogut and A. Havare, "Scattering of vector bosons by an asymmetric Hulthen potential," Journal of Physics A: Mathematical and Theoretical, vol. 43, no. 22, Article ID 225204, 225204, 14 pages, 2010.

[15] A. Arda, O. Aydogdu, and R. Sever, "Scattering and bound state solutions of the asymmetric Hulthen potential," Physica Scripta, vol. 84, Article ID 025004, 6 pages, 2011.
[16] S. Alpdoğan, O. Aydoğdu, and A. Havare, "Relativistic spinless particles in the generalized asymmetric Woods-Saxon potential," Journal of Physics A: Mathematical and Theoretical, vol. 46, no. 1, Article ID 015301, 2013.

[17] H. J. Wollersheim, H. Emling, H. Grein et al., "Coulomb excitation of ${ }^{226}$ Ra," Nuclear Physics A, vol. 556, no. 2, pp. 261280, 1993.

[18] D. T. Yordanov, D. L. Balabanski, J. Bieroń et al., "Spins, electromagnetic moments, and isomers of ${ }^{107-129} \mathrm{Cd}$," Physical Review Letters, vol. 110, no. 19, Article ID 192501, 2013.

[19] L. P. Gaffney, P. A. Butler, M. Scheck et al., "Studies of pearshaped nuclei using accelerated radioactive beams," Nature, vol. 497, no. 7448, pp. 199-204, 2013.

[20] O. Aydoğdu, A. Arda, and R. Sever, "Effective-mass Dirac equation for Woods-Saxon potential: scattering, bound states, and resonances," Journal of Mathematical Physics, vol. 53, no. 4, Article ID 042106, 2012.

[21] O. Aydoğdu, A. Arda, and R. Sever, "Scattering of a spinless particle by an asymmetric Hulthén potential within the effective mass formalism," Journal of Mathematical Physics, vol. 53, no. 10, Article ID 102111, 2012.

[22] N. Candemir and O. Bayrak, "Massive Dirac equation in asymmetric Hulthén potential," Journal of Mathematical Physics, vol. 54, no. 4, Article ID 042104, 2013.

[23] G. Bastard, Wave Mechanics Applied to Semiconductor Heterostructures, Les Ulis: Editions de Physique, 1988.

[24] F. A. de Saavedra, G. Co, A. Fabrocini, and S. Fantoni, "Model calculations of doubly closed shell nuclei in CBF theory III. j-j coupling and isospin dependence," Nuclear Physics A, vol. 605, no. 3, pp. 359-386, 1996.

[25] L. Serra and E. Lipparini, "Spin response of unpolarized quantum dots," Europhysics Letters, vol. 40, no. 6, pp. 667-672, 1997.

[26] O. Rojo and J. S. Levinger, "Integrated cross section for a velocity-dependent potential," Physical Review, vol. 123, no. 6, pp. 2177-2179, 1961.

[27] M. Razavy, G. Field, and J. S. Levinger, "Analytical solutions for velocity-dependent nuclear potentials," Physical Review, vol. 125, no. 1, pp. 269-272, 1962.

[28] R. D. Woods and D. S. Saxon, "Diffuse surface optical model for nucleon-nuclei scattering," Physical Review, vol. 95, no. 2, pp. 577-578, 1954.

[29] P. Mierzyński and K. Pomorski, "Shell structure of cesium layer covering the C fullerene core," European Physical Journal D, vol. 21, no. 3, pp. 311-314, 2002.

[30] R. C. Greenhow and J. A. D. Matthew, "Continuum computer solutions of the Schrödinger equation," Journal of Physics B, vol. 4677, 1991.

[31] A. Diaz-Torres and W. Scheid, "Two center shell model with Woods-Saxon potentials: adiabatic and diabatic states in fusion," Nuclear Physics A, vol. 757, no. 3-4, pp. 373-389, 2005.

[32] H. Erkol and E. Demiralp, "The Woods-Saxon potential with point interactions," Physics Letters A, vol. 365, no. 1-2, pp. 5563, 2007.

[33] M. Abramowitz and A. Stegun, Handbook of Mathematical Functions, Dover, New York, NY, USA, 1965. 

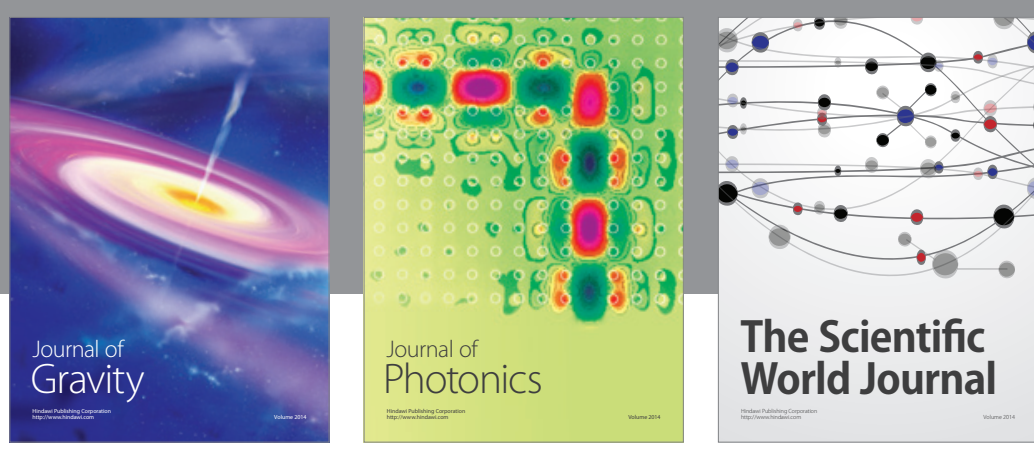

The Scientific World Journal
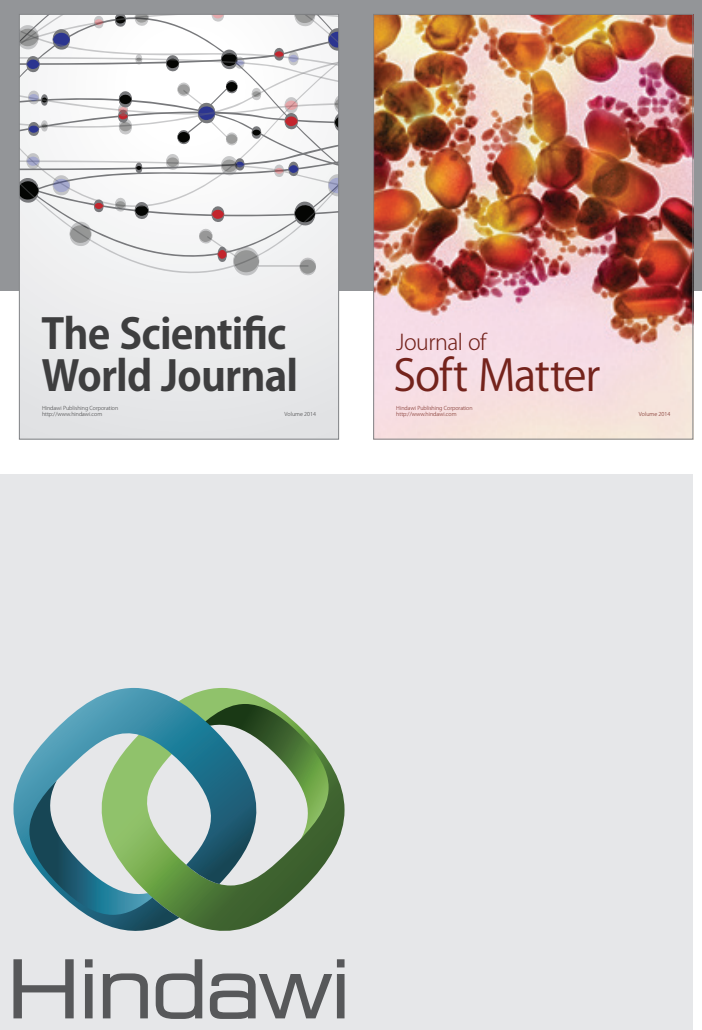

Submit your manuscripts at

http://www.hindawi.com

nternational Journal of

Statistical Mechanics
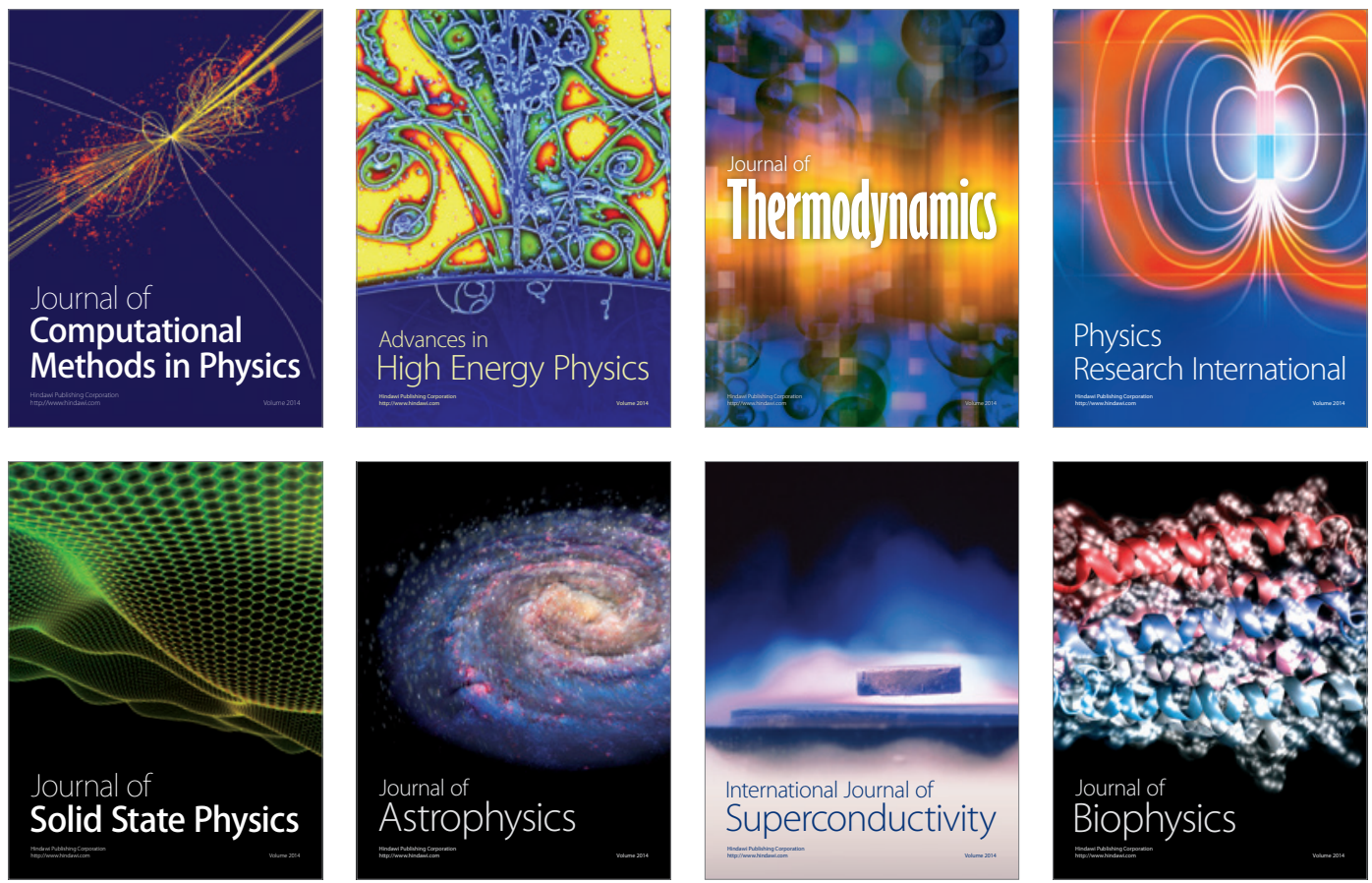
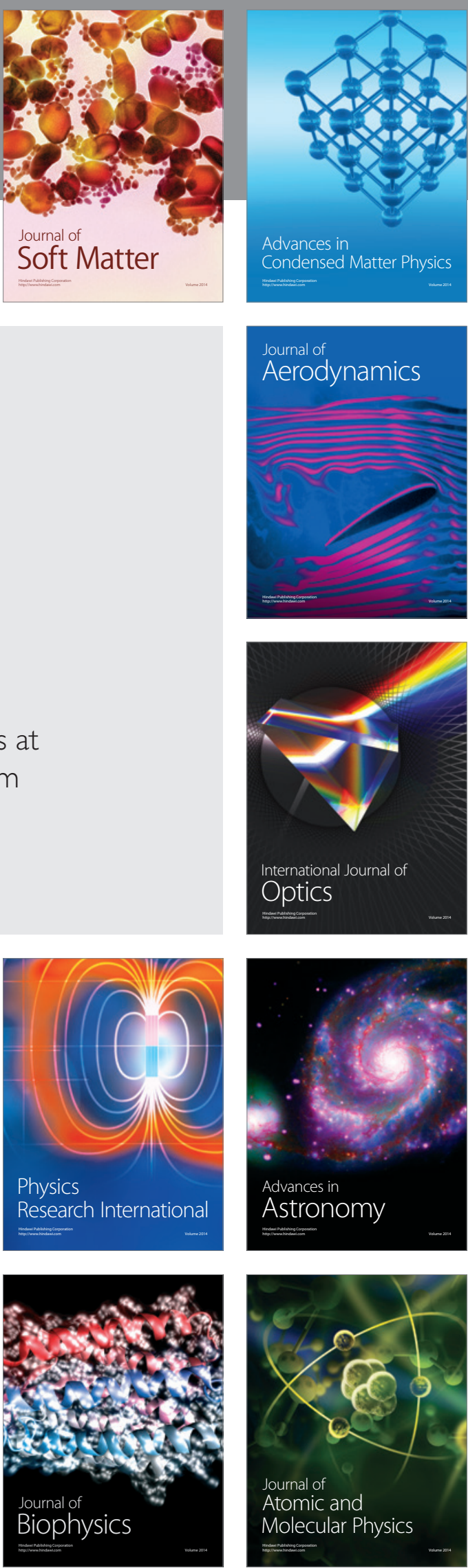